\title{
Economic Planning of Electric Vehicle Charging Stations Considering Traffic Constraints and Load Profile Templates
}

\author{
Yue Xiang ${ }^{1 *}$, Junyong $\mathrm{Liu}^{1}$, Ran $\mathrm{Li}^{2}$, Furong $\mathrm{Li}^{2}$, Chenghong $\mathrm{Gu}^{2}$, Shuoya Tang ${ }^{1}$ \\ ${ }^{1}$ School of Electrical Engineering and Information, Sichuan University, Chengdu 610065, China \\ ${ }^{2}$ Department of Electronic and Electrical Engineering, University of Bath, Bath, BA2 7AY, UK \\ *Corresponding author: exxyye@gmail.com.
}

\begin{abstract}
This paper develops a novel solution to integrate electric vehicles and optimally determine the siting and sizing of charging stations (CSs), considering the interactions between power and transportation industries. Firstly, the origin-destination (OD) traffic flow data is optimally assigned to the transportation network, which is then utilized to determine the capacity of charging stations. Secondly, the charging demand of charging infrastructures is integrated into a cost-based model to evaluate the economics of candidate plans. Furthermore, load capability constraints are proposed to evaluate whether the candidate CSs deployment and tie line plans could be adopted. Different scenarios generated by load profile templates are innovatively integrated into the economic planning model to deal with uncertain operational states. The models and framework are demonstrated and verified by a test case, which offers a perspective for effectively realizing optimal planning of the CSs considering the constraints from both transportation and distribution networks.
\end{abstract}

Key words: electric vehicle charging stations, planning, traffic flow, load capability, load profile templates.

\section{Nomenclature}

\section{Scalars and Parameters:}

$b$ is a parameter for the BPR function.

$N_{\mathrm{D}}$ is the set of buses in the distribution network.

$L_{\mathrm{D}}$ is the set of lines in the distribution network.

$N_{\mathrm{T}}$ is the set of nodes in the transportation network.

$L_{\mathrm{T}}$ is the set of links in the transportation network.

$d_{m-n}$ is the distance between CSs at node $m$ and $n$.

$d^{\text {min }}$ represents the allowed minimum distance between any CS pair.

$t_{a}^{0}$ is the free-flow travel time for $\operatorname{road} a$.

$c_{a}$ is the traffic capacity of $\operatorname{road} a$.

$b, \quad v \quad$ are the retardation factor, respectively.

$\Omega$ is the set of candidate CS nodes in the T-network.

$\Delta t$ is the predicted time interval.

\section{Variables:}

$f r_{a}$ is the traffic flow on $\operatorname{road} a$.

$t_{a}\left(f r_{a}\right)$ is the road impedance function, mainly indicating the travel time for road $a$.

$f p_{k}^{r u}$ is the traffic flow on path $k$ connecting the original-destination (OD) pair $r-u$.

$q_{r u}$ is the total traffic flow between the OD pair $r-u$.

$\delta_{a, k}^{r s}$ is the 0-1 variable reflecting whether $\operatorname{road} a$ is included in path $k$ connecting the OD pair $r$ - $u$.

$\lambda_{j, t}$ is the equivalent average arrival rate at node $j$ in time period $t$.

$f n_{j, t}$ is the traffic flow captured by the CS at node $j$ in the time period $t$, which can be obtained through the sum of the corresponding $f r_{a}$ with 
$H$ is the typical daily charging times of vehicles in the planning area.

$\omega$ is the average ratio of daily charging in CS.

$\varepsilon_{t}$ is the normalized traffic flow coefficient, in time period $t$, to reflect the daily charging ratio. $\mu$ is the average service rate of charging device.

$p_{0}$ is the free probability of the CS that EV can get charged by a charging service.

$W_{q}^{\max }$ is the maximum average waiting time.

$s^{\min }, s^{\max }$ are the lower and upper limits for the number of the charging devices in each $\mathrm{CS}$, respectively.

$r_{0} \quad$ is the interest rate.

$m_{\mathrm{C}}$ is the capital recovery coefficient for the CSs.

$M \quad$ is the total number of the scenarios.

$C_{i}^{\mathrm{CS} \_ \text {fix }}$ and $C_{i}^{\mathrm{CS} \_ \text {var }}$ are the fixed and variable investment of the CS at bus $i$, respectively.

$\delta^{\mathrm{S}}$ is the unit operation cost for substations.

$\delta^{\text {loss }}$ is the unit cost for the power loss.

$D$ is the days of the target year.

$g_{i j}$ is the conductance of line $i j$.

$\psi_{\mathrm{L}}, \psi_{C}$ are the bus sets for the lines, and the candidate CSs in the distribution network.

$P_{\mathrm{B} i}$ represents the base value for the load profile.

$N_{k, i}$ is the number of the $k_{\mathrm{th}}$ type of the classes at bus $i$.

$\sigma_{k, t, m}$ is the profile coefficient of the $k_{\text {th }}$ type classes in the time period $t$ for scenario $m$.

$P_{\mathrm{L} i, t, m}$ represents the conventional active load demand at bus $i$ in time period $t$.

$P_{i, t}^{\mathrm{CP}}$ represents the charging demand from the charging points (CPs) at bus $i$ in time period $t$.

$Q_{i, t, m}^{\mathrm{S}}$ and $Q_{\mathrm{L} i, t, m}$ are reactive power of the substation and the conventional reactive load demand at bus $i$ in time period $t$, respectively.

$V^{\min }, V^{\max }$ are the lower and upper limits for the voltage magnitude, respectively.

$P_{i j}^{\max }$ is the upper limit of the power flow at line $i j$.

$P_{i}^{\mathrm{S} \_0}$ is the capacity of the substation at bus $i$, if $x_{i}^{\mathrm{S}}=0, \quad P_{i}^{\mathrm{S} \_0}=0$.

$N_{B}$ is the total number of buses.

$N_{S}$ is the number of the substations.

$N_{L}$ is the number of lines in operation. the same injection direction.

$\rho \quad$ is the average service rate of the CS.

$S \quad$ is the number of available charging devices.

$W_{q}$ is the average waiting time.

$\beta$ is the average service rate of a charging device. It is noted that $\beta$ should be less than 1.0 , to guarantee the statistic equilibrium for the operation of the system.

$x_{i}^{\mathrm{CS}}$ is the binary variable for indicating the state of CSs in the D-network. If a candidate CS exists at bus $i$ and is included in the final solution, $x_{i}^{\mathrm{CS}}=1$, otherwise 0 .

$F \mathrm{c}$ is the total cost.

$C_{\mathrm{CS}}$ is the annual investment for CSs.

$C_{\text {sub }}$ is the annual operation cost of the substations.

$C_{\text {loss }}$ is the annual cost for the power loss.

$P_{i, t, m}^{\mathrm{S}}$ is the power output of the substation at bus $i$ in time period $t$ in the $m_{\mathrm{th}}$ scenario.

$V_{i, t, m}$ is the voltage magnitude at bus $i$ in time period $t$ in the $m_{\mathrm{th}}$ scenario.

$\theta_{i j, t, m}$ is the phase angle deviation of line $i j$ in time period $t$ in the $m_{\mathrm{th}}$ scenario.

$x_{i}^{\mathrm{S}}$ is the binary variable for indicating the state of substations. If a substation exists at bus $i$, $x_{i}^{\mathrm{S}}=1$, otherwise 0 .

$x_{i j}^{\mathrm{L}}$ is the binary variable for indicating the state of lines. If line $i j$ is in the final solution, $x_{i j}^{\mathrm{L}}=1$, otherwise 0 .

$G_{i j}\left(x_{i j}^{\mathrm{L}}\right)$ and $B_{i j}\left(x_{i j}^{\mathrm{L}}\right)$ are the real and imaginary item of the nodal admittance matrix, respectively. The matrix is closely affected and determined by the state of $x_{i j}^{\mathrm{L}}$.

$P_{i, t}^{\mathrm{CS}}$ represent the charging demand from the CSs at bus $i$ in time period $t$.

$P_{i j, t, m}$ is the power flow at line $i j$ in time period $t$ in the $m_{\mathrm{th}}$ scenario.

$x_{j}^{\mathrm{CS} \_\mathrm{T}}$ is the binary variable for indicating the state of CSs in the T-network. If $x_{j^{*}}^{\mathrm{CS}}=1(j *$ is the corresponding bus to node $j$ in the coupled network), $x_{j}^{\mathrm{CS} \_\mathrm{T}}=1$, otherwise 0.

$F_{i, j}(x)$ is the value of the $i^{\text {th }}$ objective function in the $j^{\text {th }}$ candidate plan. 


\begin{tabular}{|c|c|}
\hline $\begin{array}{l}\varpi \text { is the working efficiency of the charging } \\
\text { device }(0<\varpi \leq 1) \text {. } \\
P_{\mathrm{CD}} \text { is the charging capacity of a charging device. } \\
P_{\mathrm{CP}} \text { is the charging rate of a CP. } \\
\kappa \text { is the service ability of a CP (vehicles/day). } \\
\gamma \text { is the vacant rate }(0 \leq \gamma \leq 1) \text {. } \\
\eta_{i, t} \text { is the normalized parking demand coefficient } \\
\text { to reflect the charging demand of the CPs at } \\
\text { bus } i \text { in time period } t \text {. } \\
\tau_{i} \text { is the weight factor for the } i^{\text {th }} \\
\text { objective, if all objectives share the } \\
\text { same weight, then } \tau_{i}=1\end{array}$ & $\begin{array}{l}F_{i}\left(x^{*}\right) \text { is the best value based on the } i^{\text {th }} \text { objective } \\
\text { function. } \\
F_{i}^{\mathrm{W}} \text { is the worst value based on the } i^{\text {th }} \text { objective } \\
\text { function. } \\
N_{\mathrm{p}} \text { is the number of the available plans. } \\
F_{i, j}(x) \text { is the } i^{\text {th }} \text { objective value in the } j^{\text {th }} \text { plan. } \\
B I_{j}(x) \text { is the bargaining value for the } j^{\text {th }} \text { plan. }\end{array}$ \\
\hline
\end{tabular}

\section{Introduction}

Driven by the low carbon target, many countries have considered the electrification of transportation as one of national strategic plans and key investment areas [1-6]. As a novel distributed mobile resource, electric vehicles (EVs) are becoming a vital part for smart grid development. Charging infrastructures are the essential connection between EVs and the corresponding power system. Thus, appropriate planning of charging infrastructures is fundamental for promoting fast development of EVs while guaranteeing the normal operation of the power system. So, in this paper, how to economically plan the charging infrastructures, particularly charging stations (CSs), is studied and discussed.

Charging load estimation or forecasting is the basis for the planning of the charging infrastructures. The charging load profile was estimated in [7] by a Markov decision process, while Monte Carlo simulation based on probability distribution functions was investigated in [8]. The models were mainly from the temporal view. Moreover, a spatial-temporal model (STM) was proposed in [9] to evaluate the impact of large-scale deployment of EVs on the distribution network. The model ran based on the systematic integrations of power and transportation system analysis. An origin-destination (OD) analysis was also utilized in the model to reflect the spatial and temporal characteristics of EV charging stations. Considering multiple factors, such as oil price, social demand, battery development, etc., system dynamic and multi-agent methods were proposed in [10] to obtain more comprehensive results. The above literature provided methods for charging load estimation or forecasting from different aspects. However, not all the method could be fit for CS planning. The charging load used for planning would be aggregated by the profiles directly from EVs or reflected by other quantities, like the traffic flow, in a typical operational mode.

Moreover, the charging load should be effectively integrated with planning methods. A large volume of studies have been undertaken in planning CSs. Several Indices were proposed for selecting the site and capacity of the CSs. For example, in [11], from the view of the load density distribution, the Voronoi diagram was used for partitioning geographic areas to locate CSs and optimization models were proposed for resolving the planning problem. In [12], the criteria performance of different alternatives and criteria weights were judged by five groups of expert panels in the environmental, economic and social criteria associated with a total of sub-criteria. Indices could help make an 
assessment of the performance of the system with the charging load from the CSs in the corresponding deployment. Another common way is to build the optimal models to take various constraints into consideration to determine the best site and size for CSs. Reference [13] summarized a basic CS placement model and four potential solutions were reviewed. A mixed-integer non-linear (MINLP) optimization approach for optimal placing and sizing CSs was designed in [14]. Different station development policies were also discussed. Furthermore, customers' benefits were integrated into the models proposed in [15] and different types of charging infrastructures were considered in a social cost-based planning model [16].

As a matter of fact, since the charging behavior of EVs could be reflected by the traffic flow conditions in the transportation network, the distributions of traffic flow could be used for locating and sizing CSs. A traffic-flow capture model integrated with traffic flow data was proposed in [17] to help locate CSs. Reference [18] introduced a charging traffic flow, which contains both spatial and temporal properties of a charging load, as a discrete sequence to describe charging start events. In [19], a battery capacity-constrained EV flow capturing location model was proposed to maximize EV traffic flow.

The above literature proposed different planning models for CSs. However, most of them are investigated on the transportation network or the CS itself without considering the power supply ability of the corresponding power networks. The fact is that the increasing charging load demand would threaten the reliable operation of the coupled distribution network [20], and the network constraints from the distribution network would greatly impact the deployment of the charging infrastructures. So, the network from the corresponding power network should be also considered in the CS planning. The siting and sizing of CSs should be also integrated into the distribution network planning model. A traffic flow based siting and sizing model for CSs in the transportation network was presented in [21], and the network constraint of the distribution network was also included. An integrated planning model of CS placement was proposed in [22], and the load shift performance was considered in the model. Additionally, an economic model aiming at minimizing the total cost was proposed in [23] to achieve the coordinated planning both the reinforcement of distribution network and deployment of CSs.

It can be seen from above literatures that incorporating EV infrastructures into an existing transportation network and distribution network is challenging. Although there are plenty of studies on planning CSs, few captured the network constraints for both the power and transportation networks. Another problem for planning lies in dealing with the selection of the appropriate operational modes (scenarios). In fact, it was often solved by deterministic approaches, e.g. the typical single load profile was used to check network constraints, where the impact of operational uncertainty and variability were ignored. Usually, they were treated in an overly simplified way by planning networks for the worst case. However, it would not work appropriately in long-run planning problems because of large variability in operation that can result in added stress to the system. Thus, the uncertainty of operational states should be considered and integrated in the load capability assessment for determining the optimal CS plan.

In order to effectively deal with the uncertain operational states problem and address the economic planning for CSs from both the power and transportation perspectives, the paper proposes a novel integrated planning framework. Origin-destination (OD) analysis is characterized to benchmark the behaviors of EVs, where a System Optimization (SO) assignment model is used to obtain the equilibrium traffic flow and a 
queuing model is used to determine the sizing of CSs. Since the economic factor is the fundamental issue in planning, the designed optimal model, including the coordinated placement of CSs and the tie lines of the distribution network, mainly aims at minimizing the investment and operational cost. The load capability of the whole distribution network considering charging load is constrained and integrated into the economic planning model to reflect EV impacts on the distribution network, i.e. its ability to accommodate additional charging load demand. Representative load profile templates are introduced and used to generate different scenarios for presenting the operational uncertainties of the distribution network. Therefore, the organization and implementation of the integrated economic planning framework in the paper is presented as shown in Fig. 1.

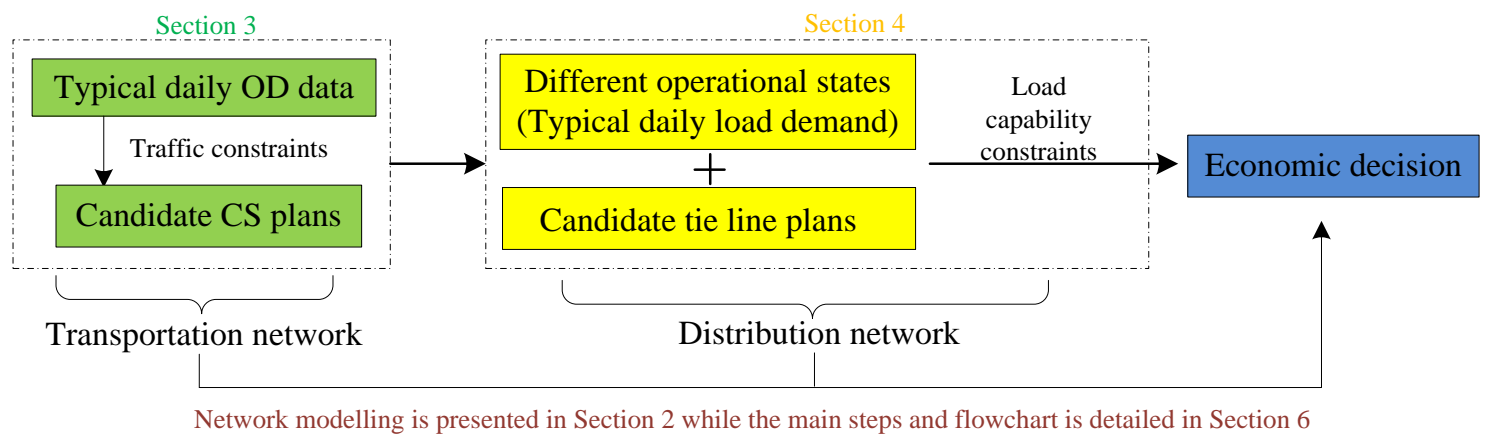

Fig. 1 Integrated economic planning framework

So, as depicted in Fig. 1, the rest of the paper are organized as follows. In Section 2, the network modelling of the coupled distribution and transportation is presented. Then, in Section 3, traffic flow is optimally assigned on each road and used in the capacity determination of candidate CSs based on a queuing model. An economic planning model is formulated in Section 4. And the load profile templates are also introduced and integrated into the model. In Section 5, the whole planning flowchart is provided. The test case is shown in Section 6 and Section 7 presents the conclusions and future work.

\section{Hierarchical network modelling}

In this paper, the transportation and distribution network are coupled and connected together according to the geographic information, as shown in Fig. 2. Let $G_{\mathrm{D}}\left(N_{\mathrm{D}}, L_{\mathrm{D}}\right)$ denote the distribution network, where $N_{\mathrm{D}}$ and $L_{\mathrm{D}}$ are the sets of buses and lines, respectively. $G_{\mathrm{T}}\left(N_{\mathrm{T}}, L_{\mathrm{T}}\right)$ denotes the transportation network in the same urban area, sharing the same geographic information with $G_{\mathrm{D}}$, where $N_{\mathrm{T}}$ and $L_{\mathrm{T}}$ are the sets of nodes and links, respectively. Assuming that travel demands originate from a set of origin nodes, and destine for a set of destinations $S \in N_{\mathrm{T}}$, which is defined as the OD pairs. 


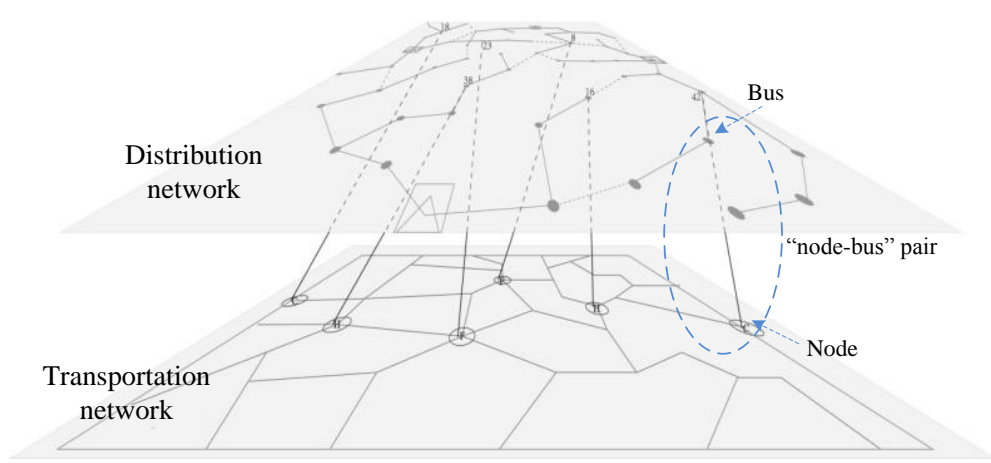

Fig. 2 The coupled networks

CSs are assumed to be located at the nodes of the transportation network, and connected to the corresponding buses in the distribution network through special electric cables, shown as the mapping "node-bus" pair in Fig. 1. In the coupled networks, the charging behavior is reflected by the traffic flow in the transportation network, and the charging is accumulated as additional load demand at the corresponding bus in the distribution network. Although there is no direct correlation between CSs, the geography distance constraint exists between any two of them, i.e. two "neighboring" CSs should not be built "too close", in order to be cost-effective. The distance between stations should meet:

$$
d_{m-n} \geq d^{\text {min }}
$$

There are several ways to determine $d_{m-n}$. If the detailed geographic road information about the area is obtained, $d_{m-n}$ can be set as the real shortest road length $(\mathrm{km})$ between two locations. Otherwise, a simplified estimated method can be applied, which uses $d_{m-n}=\xi \sqrt{\left(X_{m}-X_{n}\right)^{2}+\left(Y_{m}-Y_{n}\right)^{2}}$ to roughly present the distance, where $\left(X_{m}, Y_{m}\right)$ represents the geographical coordinate of node $m$ in the transportation network, $\xi$ is the distance modification coefficient [24].

\section{Capacity determination for charging stations}

In this part, the equilibrium traffic flow is firstly proposed and calculated by the system optimization (SO) model. Based on the assigned equilibrium traffic flow, a queuing model is then applied to determine the capacity of the CSs in the corresponding candidate plan.

\subsection{Traffic flow assignment}

Since the EV is used for its owner's transportation demand, the behavior of the EV is determined by the owner's habit and trip demand, and thus, any study related to EVs would be unrealistic without transportation features. The mobile and transportation features of EVs are reflected by CSs. As described in the paper, the "node" of the transportation network can be regarded as the candidate location for CS construction. The siting of CSs would reflect the charging demand, and the traffic flow can indicate the aggregated degree of the EVs passing by the node. A larger value is assumed to reflect larger probability that the EVs need to be conveniently charged. So, in this way, the traffic information can be used to simulate the charging behavior and help estimate the charging demand, and the planning of the CSs further. Generally, navigation systems, 
like BaiduMap API [25], could provide a large volume of real-time or historical data of traffic flows. However, the raw dynamic traffic flow data cannot be used directly in the CS planning. Thus, typical daily OD data are utilized to generate typical traffic flow for planning. In order to achieve that, SO model [26] is used to generate and assign the traffic flow for each road of a transportation network. The objective of the SO model is to achieve minimum traveling cost:

$$
\begin{gathered}
\min \sum_{a \in N_{T}} f r_{a} t_{a}\left(f r_{a}\right) \\
\text { s.t. } \sum_{k} f p_{k}^{r u}=q_{r u}, \quad f p_{k}^{r u} \geq 0 \\
f r_{a}=\sum_{r} \sum_{u} \sum_{k} f p_{k}^{r u} \delta_{a, k}^{r u} \\
t_{a}\left(f r_{a}\right)=t_{a}^{0}\left[1+b\left(\frac{f r_{a}}{c_{a}}\right)^{v}\right]
\end{gathered}
$$

It is assumed that the travel time $t_{a}$ for $\operatorname{road} a$ is a strictly increasing function with respect to the aggregated flow on the road, i.e. $t_{a}\left(f r_{a}\right)$. Thus, the Bureau of Public Roads (BPR) function [21] shown in Eq. (5), is used. The assigned traffic flow based on the SO model is defined as "equilibrium traffic flow" in this paper. A detailed description of the SO model can be found in [26].

\subsection{Capacity determination based on queuing model}

After the equilibrium traffic flow of each road is obtained, queuing theory is used to analyze the mobility and randomness of EVs and help determine the capacity of the CSs, i.e. the number of the charging devices.

The service system of CSs can be regarded as an M/M/S queuing system, and thus the customers of the queuing system are represented by EVs, where reception corresponds to the charging devices and providing service means charging. In reality, the arrival time of each EV at a CS is random, but it may follow certain distributions in some time periods according to the statistic studied. Thus, in the paper, the arrival of EV in a certain CS is described as a Poisson process while the interval time of arriving obeys negative exponent distribution [27].

According to the characteristics of Poisson process, an important parameter, which is the average arrival rate, i.e. $\lambda$, represents the number of occurring random events in unit time in theory. It indicates the average number of EVs arriving CSs in a time segment. Integrated with the generated traffic flow, it can be formulated as:

$$
\lambda_{j, t}=H \omega \varepsilon_{t} \frac{f n_{j, t}}{\sum_{j \in \Omega} f n_{j, t}} / \Delta t
$$

where $H$ is the total daily charging times of vehicles in the CSs of the planning area within time horizon $T$, which could be predicted according to several factors, like EV scale in the area, statistical EV proportion, charging frequency etc. [28].

Then, the performance indices of the service system, i.e. a certain CS, can be obtained based on the queuing model [27] and formulated as:

$$
\rho=\frac{\lambda}{\mu}
$$




$$
\begin{gathered}
\beta=\frac{\lambda}{s \mu} \\
p_{0}=1 /\left[\sum_{n=0}^{s-1} \frac{\rho^{n}}{n !}+\frac{\rho^{s}}{s !} \frac{1}{1-\beta}\right] \\
W_{q}=\frac{s \rho^{s+1} p_{0}}{\lambda s !(s-\rho)^{2}}
\end{gathered}
$$

In theory, more charging devices mean more investment, which however is not economic in planning. Thus, $W_{q}$ is used to indicate customers' patience for charging in a $\mathrm{CS}$, and help deploy charging devices. In another way, if the waiting time of a customer is over a certain period, they will leave, i.e. $W_{q} \leq W_{q}^{\max }$ is used to determine the number of charging devices. Generally, it is difficult to obtain the inverse functions of Eq. (10) and solve the appropriate result directly, and thus an enumeration method is used. For the candidate CS at node $j$ according to the maximum $\lambda_{j}$ among time periods, we can initialize $s$, and try to increase it by plus 1 in each step, calculate $W_{q}$ and compare it with the given $W_{q}^{\max }$, till $W_{q} \leq W_{q}^{\max }$. Then the corresponding $s$ would be the cost-effective number of charging devices.

In sizing process, the number of charging devices in a possible location should also satisfy:

$$
s^{\min } x_{i}^{\mathrm{CS}} \leq s_{i} x_{i}^{\mathrm{CS}} \leq s^{\max } x_{i}^{\mathrm{CS}}
$$

where $s^{\min }, s^{\max }$ are the lower and upper limits for the number of charging devices in each CS respectively.

\section{Economic planning model}

4.1 Economic objectives

An economic model is designed for the planning. The cost in the target year can be optimized by comparing a suitable set of alternative plans using the following economic model, which includes investment and operational cost:

$$
\begin{gathered}
\min F_{\mathrm{C}}=C_{\mathrm{CS}}+C_{\text {sub }}+C_{\text {loss }} \\
C_{\mathrm{CS}}=C_{\mathrm{CS} \_f i x}+C_{\mathrm{CS} \_v a r}=\frac{r_{0}\left(1+r_{0}\right)^{m_{\mathrm{C}}}}{\left(1+r_{0}\right)^{m_{\mathrm{C}}}-1} \sum_{i \in \psi_{C}} x_{i}^{\mathrm{CS}}\left(C_{i}^{\mathrm{CS} \_\mathrm{fix}}+s_{i} C_{i}^{\mathrm{CS} \_v a r}\right) \\
C_{\text {sub }}=D \sum_{m=1}^{M} \rho_{m}\left(\delta^{\mathrm{S}} \sum_{t \in T} \sum_{i \in \psi_{\mathrm{S}}} P_{i, t, m}^{\mathrm{S}} x_{i}^{\mathrm{S}}\right) \\
C_{\text {loss }}=D \sum_{m=1}^{M} \rho_{m}\left(\delta^{\operatorname{loss}} \sum_{t \in T} \sum_{(i j) \in \psi_{\mathrm{L}}} x_{i j}^{\mathrm{L}} g_{i j}\left(V_{i, t, m}^{2}+V_{j, t, m}^{2}-2 V_{i, t, m} V_{j, t, m} \cos \theta_{i j, t, m}\right)\right)
\end{gathered}
$$

where $F_{\mathrm{c}}$ is the total cost, including three parts: $C_{\mathrm{CS}}$ is the annual investment for CSs; $C_{\text {sub }}$ is the annual operation cost of the substations; $C_{\text {loss }}$ is the annual cost for the power loss. $\psi_{\mathrm{L}}, \psi_{C}$ are the bus sets of the lines and the candidate CSs. $x_{i}^{\mathrm{S}}, x_{i j}^{\mathrm{L}}, x_{i}^{\mathrm{CS}}$ are the binary variables for indicating the state of substation buses, lines and CSs. If a 
substation exists at bus $i, x_{i}^{\mathrm{S}}=1$, otherwise 0 . If a line is in operation in the final solution, $x_{i j}^{\mathrm{L}}=1$, otherwise 0 . If a candidate CS exists at bus $i$ and is included in the final solution, $x_{i}^{\mathrm{CS}}=1$, otherwise 0 . It is noted that except for the cost type in the objective function, other elements like maintenance cost of the transmission lines, etc., are almost the same in each candidate plan, so they would not affect the final results and thus are not added into the optimal formula.

\subsection{Load templates and scenarios}

The planning of CSs needs to take care of the uncertain operational states of the distribution network. Scenarios are utilized to reflect a very limited number of but typical operational states in this paper, which can be aggregated and generated by customers' load profiles. Due to the diversity of customer types, it is impractical to collect the load profile of every customer continuously over time [29]. Thus, representative load profile templates can be clustered to reflect the detailed features of each customer through clustering technology [30]. For example, in the UK, the Electricity Association has studied loads in England and set about a program of analyzes in order to define the number and types of profiles to be used in the settlement, which leads to eight generic profile classes representing a large population of similar customers [31]. In Norway, the Norwegian Water Resources and Energy Administration also developed the standard load profiles for unmetered customers [29]. According to different energy utilization habit, particular load profile templates could be produced in different countries or areas.

With the available load templates of the target area, the aggregated load profiles in different scenarios will be obtained using the templates and other information, and the aggregated load profile at bus $i$ can be formulated:

$$
P_{\mathrm{L} i, t, m}=\sum_{k=1}^{K} P_{\mathrm{B} i} \sigma_{k, t, m} N_{k, i}
$$

\subsection{Load capability constraints}

The charging demand from charging infrastructures should satisfy the capacity of the distribution network, i.e. load capability constraints. In this paper, the load capability can be determined by two elements: one is network topology, which is to say that different network configuration plans contribute to a different quantification combination of $x_{i j}^{\mathrm{L}}$, that will affect the capability to adopt charging infrastructures; another is the basic network and operation constraints. Without considering distribution network reinforcement, different network configurations, i.e. tie lines setting, as well as different operational states, i.e. load profiles, will contribute to the different load capability of the system. The main load capability constraints are listed as follows:

a. Power balance equations:

$$
\begin{gathered}
P_{i, t, m}^{\mathrm{S}} x_{i}^{\mathrm{S}}-P_{\mathrm{Li}, t, m}-P_{i, t}^{\mathrm{CP}}-P_{i, t}^{\mathrm{CS}} x_{i}^{\mathrm{CS}}=V_{i, t, m} \sum_{j \in N_{D}} V_{j, t, m}\left(G_{i j}\left(x_{i j}^{\mathrm{L}}\right) \cos \theta_{i j, t, m}+B_{i j}\left(x_{i j}^{\mathrm{L}}\right) \sin \theta_{i j, t, m}\right) \\
Q_{i, t, m}^{\mathrm{S}} x_{i}^{\mathrm{S}}-Q_{\mathrm{L} i, t, m}=V_{i, t, m} \sum_{j \in N_{D}} V_{j, t, m}\left(G_{i j}\left(x_{i j}^{\mathrm{L}}\right) \sin \theta_{i j, t, m}-B_{i j}\left(x_{i j}^{\mathrm{L}}\right) \cos \theta_{i j, t, m}\right)
\end{gathered}
$$

b. Voltage magnitude:

$$
V^{\min } \leq V_{i, t, m} \leq V^{\max }
$$

c. Power flow of the lines: 


$$
\left|P_{i j, t, m}\right| \leq P_{i j}^{\max }
$$

d. Power output for the substations

$$
0 \leq P_{i, t, m}^{\mathrm{S}} \leq P_{i}^{\mathrm{S}}{ }^{0} x_{i}^{\mathrm{S}}
$$

e. Radial topology in operation

It is noted that the reinforcement of the distribution network itself is not considered in the planning, i.e. no new lines added or substation expansion. However, the status of the tie lines should be determined, to be fit for the placement of CSs with the best objective performance, and satisfy:

$$
N_{B}-N_{S}=N_{L}
$$

Equations (17), (18), (22) together make up the conditions of radiality constraint for stable operation of the distribution network without no isolated island.

\section{Planning flowchart}

The main steps for the whole planning are listed as follows:

1) Collect traffic OD data, geographic data, and assign traffic flow according to the SO model. In this way, the equilibrium traffic flow data could be obtained.

2) Assume the total number of CSs are given in the first place, then according to Eq. (1) and the geographic information, potential siting combination in the transportation network could be obtained from candidate locations.

3) Based on the queuing model formulated in Eqs. (6) (11), the minimum deployment of charging devices corresponding to the candidate sitting plans would be calculated according to the maximum waiting time. In that way, the capacity (sizing) of the corresponding CSs in each plan can be determined.

From the view of distribution network, the charging demand from the CSs at bus $j$ in time period $t$ in each candidate plans can be estimated based on:

$$
P_{j, t}^{\mathrm{CS}}=s_{j} P_{\mathrm{CD}} \varpi \beta_{j, t}
$$

Regarding to CPs, they are distributed in residential or office areas, stores or bus parking slots, and it is assumed aggregated CPs are deployed at each bus. Thus, the charging demand from the CPs $P_{i, t}^{\mathrm{CP}}$ would be estimated based on the aggregated load profiles at the corresponding bus, as formulated:

$$
N_{i}^{\mathrm{CP}}=\left[\begin{array}{c}
H \times(1-\omega) \times \sum_{m=1}^{M} \sum_{t=1}^{T} \rho_{m} P_{\mathrm{L} i, t, m} \\
\kappa \times(1-\gamma) \times \sum_{i=1}^{N_{\mathrm{D}}} \sum_{m=1}^{M} \sum_{t=1}^{T} \rho_{m} P_{\mathrm{L} i, t, m}
\end{array}\right]
$$

where $N_{i}^{\mathrm{CP}}$ is the estimated number of CPs at bus $i$.

4) Then the economic planning model is applied to select the optimal plan adapting ten scenarios. The possible network topology caused by different tie line plans in the operation of the distribution network are generated based on the branch exchange algorithm [33], indicating the possible allocation of real norm-open tie lines to meet the radial operation with no islands. The "tie lines" in reality are used for power transfer when the distribution network suffers faults. In the general operation states, the breaker on the tie lines is norm-open, that is to say, no power flow will pass. In that way, the radial operation constraint of the distribution network could be satisfied. In this case, the 
candidate tie lines need to be classified in the optimal result that some of them would be the real tie lines with norm-open breakers, while the other would be regarded as the transmission lines with norm-close breakers. That would be dependent on the economic model in Eq. (12), and the radiality constraints satisfying every operational states or scenarios.

Load capability constraints are checked in the optimization. The solution satisfying all the constraints with the lowest cost will be the final optimal plan, including the siting and sizing of CSs, as well as tie lines allocation for the distribution network.

The whole planning flowchart is shown in Fig. 3.

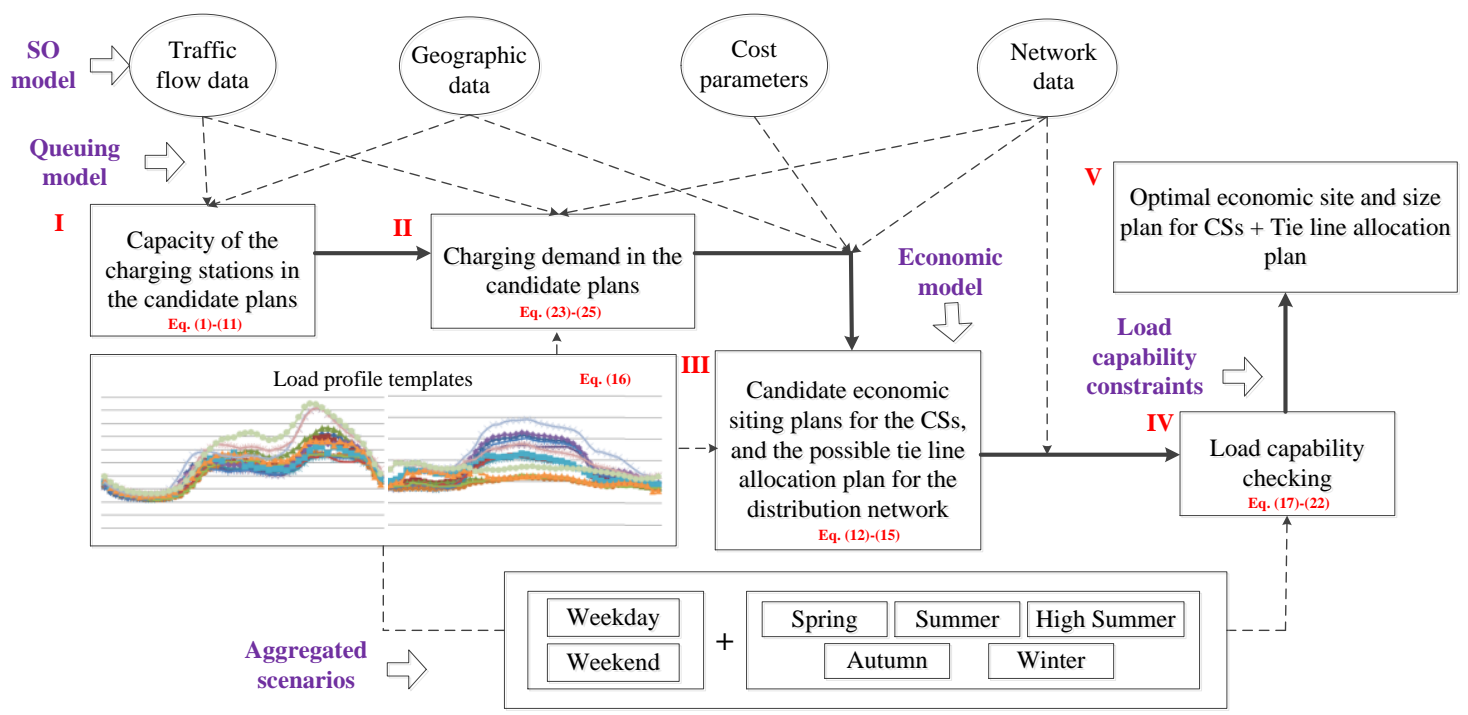

Fig. 3 Planning flowchart

\section{Case study}

\subsection{Case description}

The load templates of the UK [33] is adopted in the test case. Typical profiles of the eight classes (weekday or weekend in spring/ summer/ hot summer/ autumn/ winter) are shown in Fig. 4, and $K=8$. Class 1 2 correspond to the household type load, while class $3 \sim 5$ are the small and medium enterprise type, and class 6 to 8 are industrial ones. 


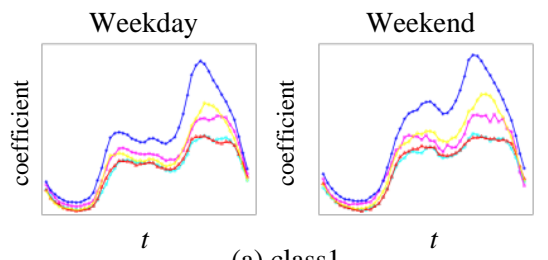

(a) class 1

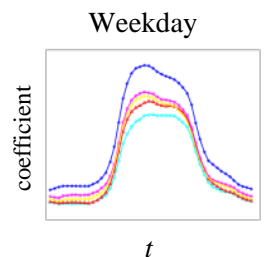

(c) class 3
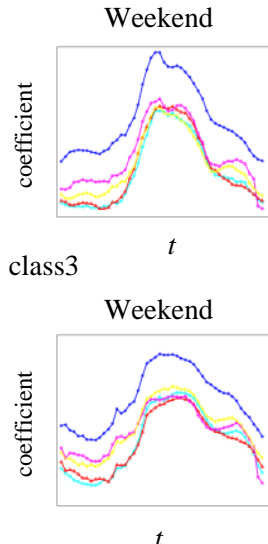

(e) class5
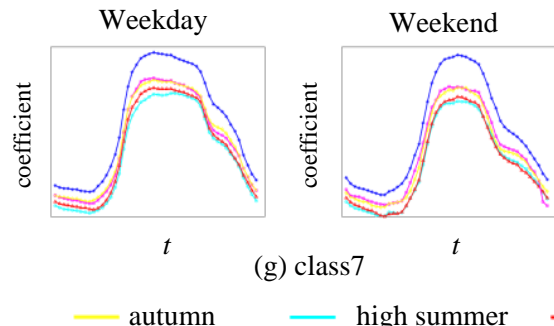

_ high summer

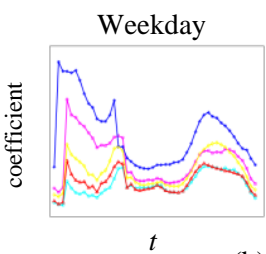

(b) class 2
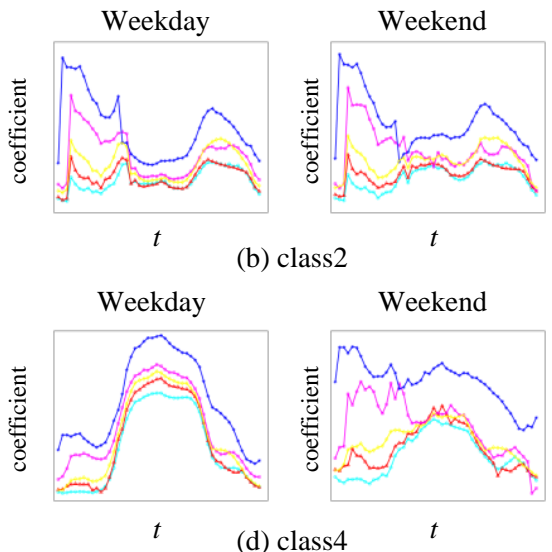

(d) class 4
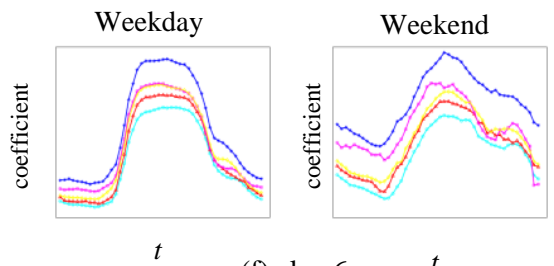

(f) class6 $t$
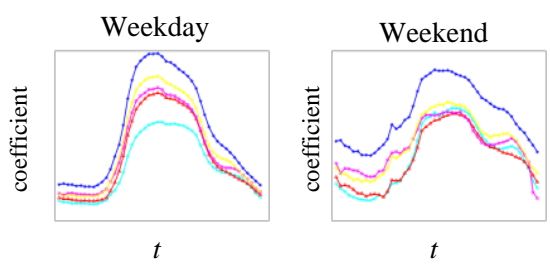

(h) class 8

summer - spring w winter

Fig. 4 Load profile classes and templates

Sioux Falls transportation network [34] is used as the test case, which contains 24 nodes and bi-direction roads are connected between neighboring nodes. $12.66 \mathrm{kV}$ 33-bus distribution network [35] is used as the corresponding power grid. Detailed line parameters can be found in Table 1. As shown in Fig. 5 (a), the links between the two layers indicate the candidate locations for the CSs, which also capture the interactions between the distribution and transportation networks. The corresponding "node-bus" pairs are listed in Table 2, also as the candidate locations for the CSs. The transportation network is shown in Fig. 5 (b). Fig. 5 (c) shows the 33-bus distribution network, where the yellow stars are the substations (10 MW each), while line \# 9, 10, 12, 14, 15, 20, 21 are the candidate tie lines whose allocation need be also determined in the optimization to guarantee the secure and radial operation with charging load demand added. Assuming there are 10000 customers or households in the urban area. Their load classes and the corresponding number are also given in Fig. 5 (c).

Based on the load profile templates, ten scenarios are generated for the planning, made up of combinations according to different types of seasons and days, e.g., spring weekday, spring weekend, summer weekday, summer weekend, high summer weekday, high summer weekend, autumn weekday, autumn weekend, winter weekday, winter weekend. 


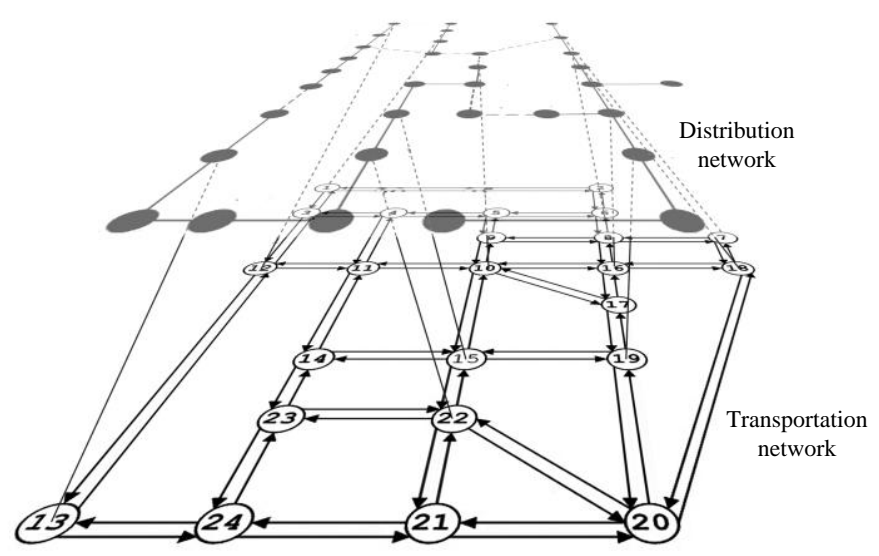

(a) The coupled networks

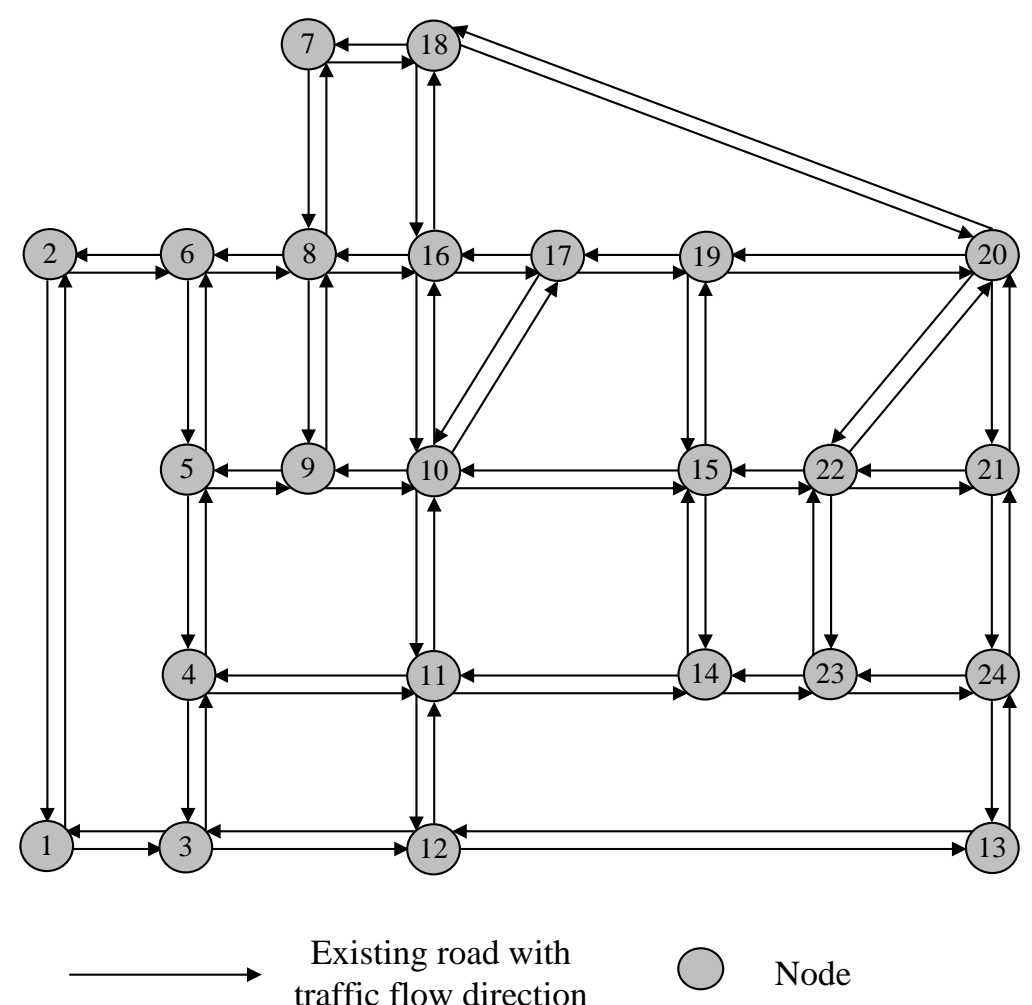

(b) Transportation network 


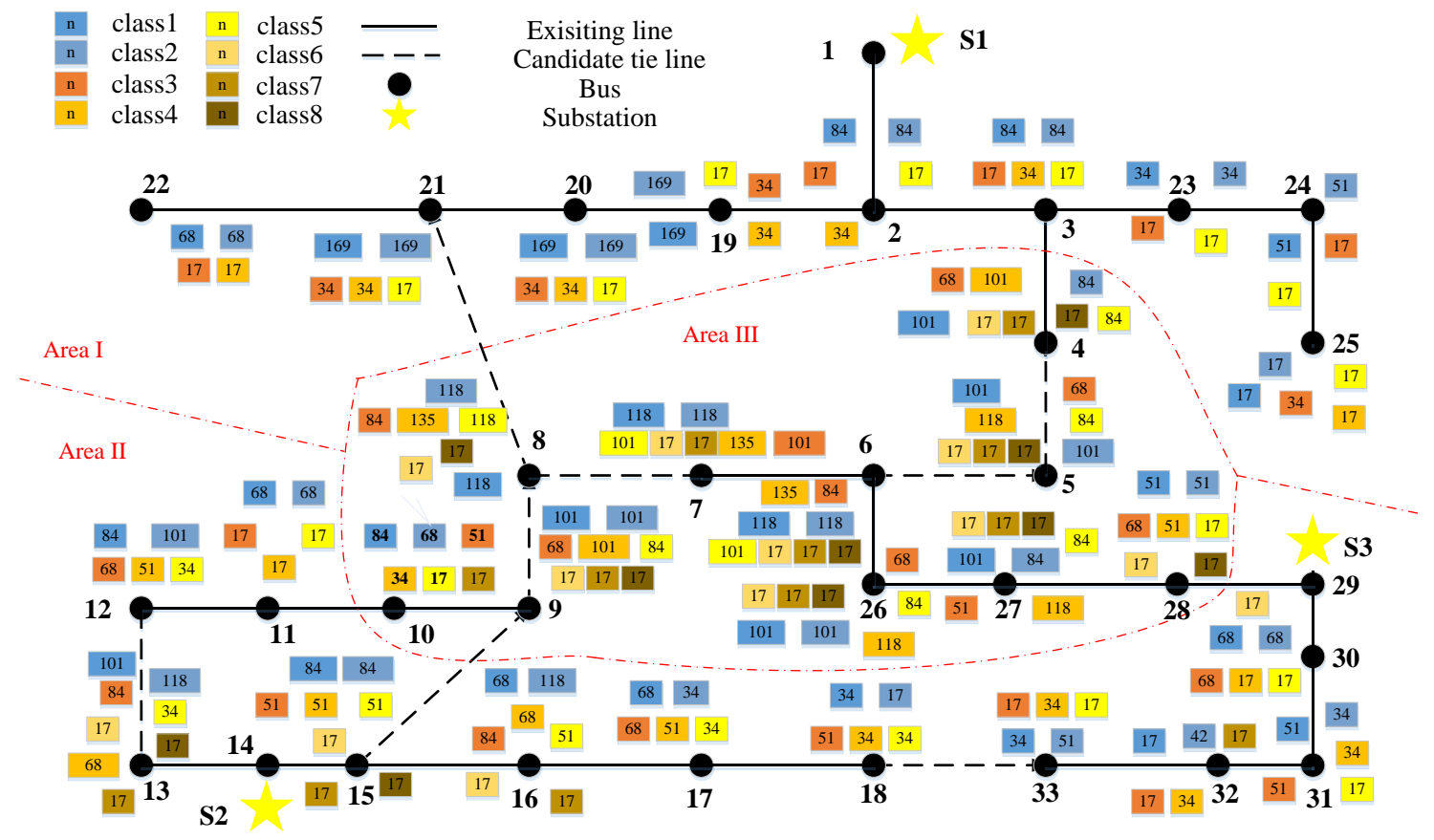

(c) Distribution network and customers' distribution with the average amount of the corresponding classes

Fig. 5 Test system

Table 1 Line parameters of the 33-bus distribution network

\begin{tabular}{ccccc|ccccc}
\hline $\begin{array}{c}\text { Line } \\
\#\end{array}$ & $\begin{array}{c}\text { Starting } \\
\text { bus }\end{array}$ & $\begin{array}{c}\text { Ending } \\
\text { bus }\end{array}$ & Resistance & Reactance & $\begin{array}{c}\text { Line } \\
\#\end{array}$ & $\begin{array}{c}\text { Starting } \\
\text { bus }\end{array}$ & $\begin{array}{c}\text { Ending } \\
\text { bus }\end{array}$ & Resistance & Reactance \\
\hline 1 & 1 & 2 & 0.0922 & 0.047 & 19 & 9 & 10 & 1.044 & 0.74 \\
2 & 21 & 22 & 0.7089 & 0.9373 & 20 & 12 & 13 & 1.468 & 1.155 \\
3 & 20 & 21 & 0.4095 & 0.4784 & 21 & 9 & 15 & 2 & 2 \\
4 & 19 & 20 & 1.5042 & 1.3554 & 22 & 13 & 14 & 0.5416 & 0.7129 \\
5 & 2 & 19 & 0.164 & 0.1565 & 23 & 14 & 15 & 0.591 & 0.526 \\
6 & 2 & 3 & 0.493 & 0.2511 & 24 & 15 & 16 & 0.7463 & 0.545 \\
7 & 3 & 23 & 0.4512 & 0.3083 & 25 & 16 & 17 & 1.289 & 1.721 \\
8 & 23 & 24 & 0.898 & 0.7091 & 26 & 17 & 18 & 0.732 & 0.574 \\
9 & 8 & 21 & 2 & 2 & 27 & 18 & 33 & 0.5 & 0.5 \\
10 & 7 & 8 & 0.7114 & 0.2351 & 28 & 32 & 33 & 0.341 & 0.5302 \\
11 & 6 & 7 & 0.1872 & 0.6188 & 29 & 31 & 32 & 0.3105 & 0.3619 \\
12 & 5 & 6 & 0.819 & 0.707 & 30 & 26 & 27 & 0.2842 & 0.1447 \\
13 & 3 & 4 & 0.366 & 0.1864 & 31 & 27 & 28 & 1.059 & 0.9337 \\
14 & 4 & 5 & 0.3811 & 0.1941 & 32 & 28 & 29 & 0.8042 & 0.7006 \\
15 & 8 & 9 & 1.03 & 0.74 & 33 & 30 & 31 & 0.9744 & 0.963 \\
16 & 6 & 26 & 0.203 & 0.1034 & 34 & 29 & 30 & 0.5075 & 0.2585 \\
17 & 11 & 12 & 0.3744 & 0.1238 & 35 & 24 & 25 & 0.896 & 0.7011 \\
18 & 10 & 11 & 0.1966 & 0.065 & & & & & \\
\hline
\end{tabular}

Table 2 "Bus-node" pair

\begin{tabular}{cc|cc|cc|cc}
\hline Node & Bus & Node & Bus & Node & Bus & Node & Bus \\
\hline 7 & 21 & 8 & 20 & 10 & 7 & 11 & 11 \\
12 & 13 & 13 & 32 & 15 & 27 & 16 & 3 \\
18 & 19 & 19 & 23 & 22 & 28 & & \\
\hline
\end{tabular}


The main parameters are set as follows: the vehicle per household is set as 1.86 [36], while the charging frequency of a vehicle is 0.65 per day [1], since there are about 10000 customers or households in the urban area, so $H=3600$. Let the maximum waiting time be $10 \mathrm{~min}$. The base value for the load profile is set as $0.15 \mathrm{~kW}$ with power factor 0.9 and the charging rate of a $\mathrm{CP}$ is set as $2.2 \mathrm{~kW}$ while that of a charging devices is 30 $\mathrm{kW} . \quad r_{0}=0.1, m_{s}=10, \omega=0.2, \varpi=1, b=0.15, \quad v=1, \rho_{m}=0.1, d^{\min }=10 \mathrm{~km}, \quad M=10$, $\delta^{\text {loss }}=50 \mathrm{USD} / \mathrm{MWh}, \delta^{\mathrm{s}}=50 \mathrm{USD} / \mathrm{MWh}$, the fixed investment for each $\mathrm{CS}$ at the location of the "bus-node" pairs are $35,27,45,38,25,20,40,45,45,35,35 \times 10^{4}$ USD, the variable cost is $11.5,10.7,12.5,11.8,10.5,10,12,12.5,12.5,11.5,12.5$ $\times 10^{4}$ USD per charging device. Let the allowable voltage drop be $10 \%$. Detailed daily trip OD data and coordinates can be found in [33], in which the link lengths of the road network are set and scaled by $10^{-4}(\mathrm{~km})$ based on the given node coordinates. The unit time period for the operation of the coupled network is set as $1 \mathrm{~h}$. The normalized parking demand coefficient for different areas and traffic flow coefficient are given in Fig. 6 and Fig. 7 according to [37] and [21]. Programs are implemented in the MATLAB environment using Celeron E3300 2.5 GHz/1.96 GB computers.

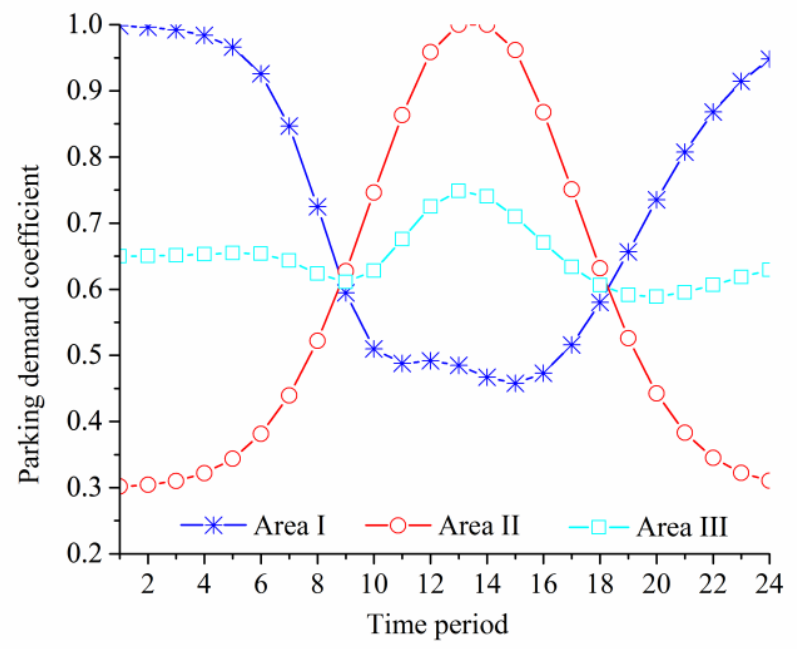

Fig. 6 Normalized parking demand coefficient for different areas

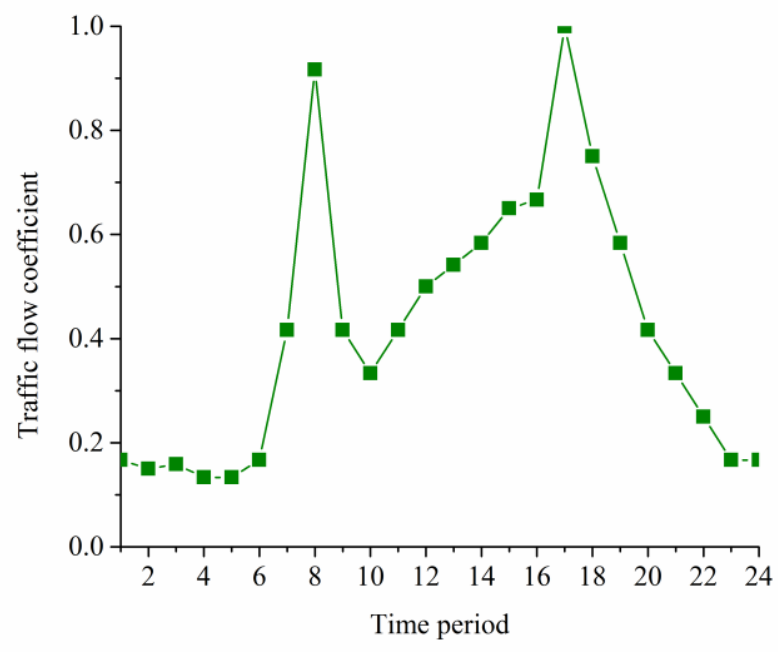

Fig. 7 Normalized hour traffic flow coefficient 


\subsection{Case results}

According to the typical traffic OD data of the 24-node transportation network from [34], the equilibrium traffic flow distribution in each time period can be obtained. Some of them could be seen in Fig. 8. Deeper color indicates heavier traffic flow on the certain road.

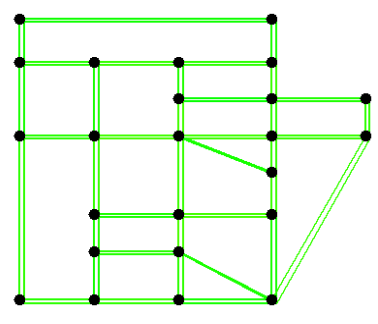

$t=1,6,23$

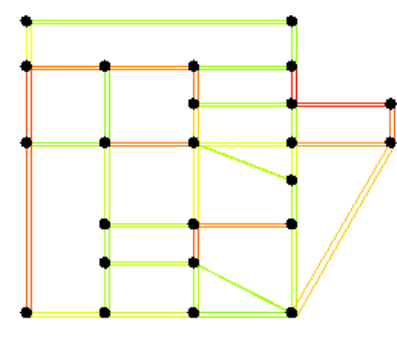

$t=17$

Fig. 8 Traffic flow profile in some time periods

The arrival rate in each time period can be then obtained based on Eq. (6), and the candidate locations for the CSs and the corresponding charging devices are as shown in Table 3, i.e. 19 possible siting and sizing plans for the CSs could be generated, based on Eqs. (1)-(11).

Table 3 Candidate siting and sizing (number of charging devices) plans for the CSs

\begin{tabular}{c|ccccccccccc}
\hline CS & Node & Node & Node & Node & Node & Node & Node & Node & Node & Node & Node \\
plan \# & 7 & 8 & 10 & 11 & 12 & 13 & 15 & 16 & 18 & 19 & 22 \\
\hline 1 & 7 & 10 & 0 & 9 & 0 & 7 & 0 & 0 & 0 & 8 & 0 \\
2 & 7 & 9 & 0 & 9 & 0 & 7 & 0 & 0 & 0 & 0 & 9 \\
3 & 7 & 9 & 0 & 9 & 0 & 0 & 0 & 0 & 0 & 8 & 9 \\
4 & 7 & 10 & 0 & 0 & 9 & 7 & 0 & 0 & 0 & 8 & 0 \\
5 & 7 & 10 & 0 & 0 & 9 & 7 & 0 & 0 & 0 & 0 & 9 \\
6 & 7 & 9 & 0 & 0 & 9 & 0 & 0 & 0 & 0 & 8 & 9 \\
7 & 7 & 10 & 0 & 0 & 0 & 7 & 0 & 0 & 0 & 8 & 9 \\
8 & 7 & 0 & 0 & 9 & 0 & 6 & 0 & 10 & 0 & 0 & 9 \\
9 & 7 & 0 & 0 & 9 & 0 & 7 & 0 & 0 & 0 & 8 & 10 \\
10 & 7 & 0 & 0 & 9 & 0 & 0 & 0 & 10 & 0 & 8 & 9 \\
11 & 7 & 0 & 0 & 0 & 9 & 6 & 0 & 10 & 0 & 0 & 9 \\
12 & 7 & 0 & 0 & 0 & 9 & 7 & 0 & 0 & 0 & 8 & 10 \\
13 & 7 & 0 & 0 & 0 & 8 & 0 & 0 & 10 & 0 & 8 & 9 \\
14 & 0 & 9 & 0 & 9 & 0 & 6 & 0 & 0 & 0 & 8 & 9 \\
15 & 0 & 9 & 0 & 0 & 9 & 6 & 0 & 0 & 0 & 8 & 9 \\
16 & 0 & 0 & 0 & 9 & 0 & 6 & 0 & 10 & 0 & 8 & 9 \\
17 & 0 & 0 & 0 & 8 & 0 & 0 & 0 & 9 & 10 & 7 & 8 \\
18 & 0 & 0 & 0 & 0 & 8 & 6 & 0 & 10 & 0 & 8 & 9 \\
19 & 0 & 0 & 0 & 0 & 7 & 0 & 0 & 9 & 10 & 7 & 8 \\
\hline
\end{tabular}

The capacity of CSs in different plans produces different charging load demand to the corresponding distribution network. In order to guarantee radial topology in operation, 6 possible network topologies, which indicates the corresponding allocation of the tie line, could be generated, as shown in Fig. 9.

However, not all of the topologies are fit for the CS plans integrated with the load capability constraints. After power flow and constraint verification in every scenario, 12 available combination plans (site and size for CSs and allocation for tie lines) pass the load capability checking, and the detailed results can be seen in Table 4. 

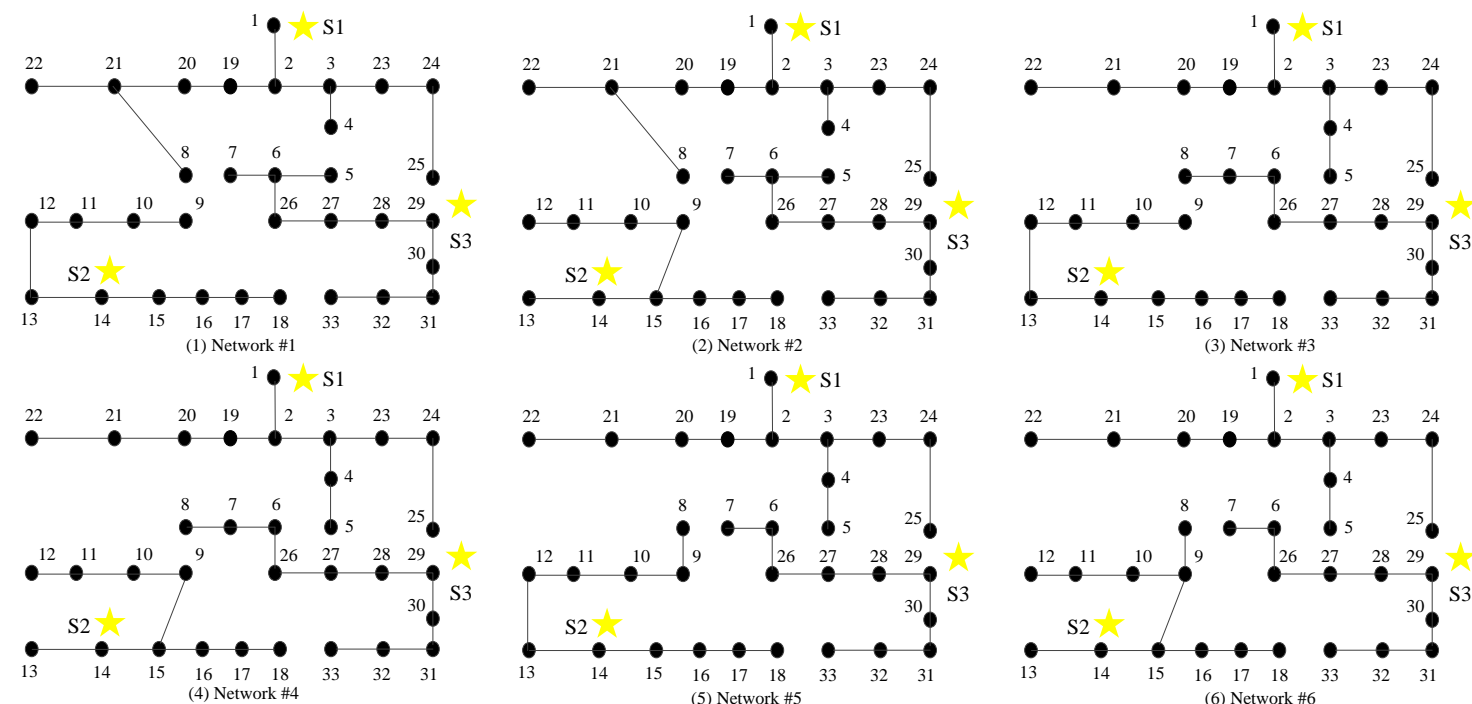

1 - S1
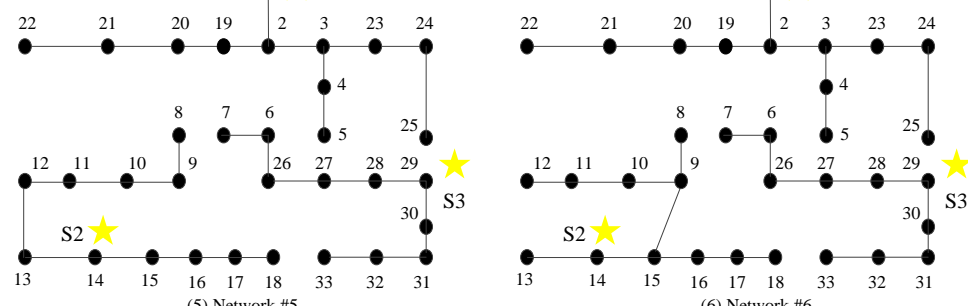

Fig. 9 Possible network topology caused by different tie line allocation

Table 4 Total cost and the constitution of the available plans

\begin{tabular}{|c|c|c|c|c|c|c|}
\hline Available plan \# & Network \# & CS plan \# & $\begin{array}{c}F_{\mathrm{C}} \\
\left(\times 10^{6} \mathrm{USD}\right)\end{array}$ & $\begin{array}{c}C_{\mathrm{CS}} \\
\left(\times 10^{6} \mathrm{USD}\right)\end{array}$ & $\begin{array}{c}C_{\text {sub }} \\
\left(\times 10^{6} \mathrm{USD}\right)\end{array}$ & $\begin{array}{c}C_{\text {loss }} \\
\left(\times 10^{5} \mathrm{USD}\right)\end{array}$ \\
\hline 1 & 5 & 7 & 5.6415 & 1.4272 & 4.1019 & 1.1245 \\
\hline 2 & 6 & 4 & 5.6126 & 1.3979 & 4.1021 & 1.1262 \\
\hline 3 & 6 & 5 & 5.6499 & 1.4323 & 4.1036 & 1.1412 \\
\hline 4 & 6 & 6 & 5.6686 & 1.4507 & 4.1037 & 1.1425 \\
\hline 5 & 6 & 7 & 5.6464 & 1.4272 & 4.1043 & 1.1489 \\
\hline 6 & 6 & 11 & 5.6616 & 1.4443 & 4.1034 & 1.1394 \\
\hline 7 & 6 & 12 & 5.6449 & 1.4272 & 4.1036 & 1.1414 \\
\hline 8 & 6 & 13 & 5.6981 & 1.4802 & 4.1036 & 1.1418 \\
\hline 9 & 6 & 15 & 5.6337 & 1.4163 & 4.1034 & 1.1398 \\
\hline 10 & 6 & 17 & 5.7373 & 1.5102 & 4.1082 & 1.1881 \\
\hline 11 & 6 & 18 & 5.6636 & 1.4459 & 4.1036 & 1.1413 \\
\hline 12 & 6 & 19 & 5.6913 & 1.4752 & 4.1028 & 1.1331 \\
\hline
\end{tabular}

According to the economic planning model, the available plan \#2 with the minimum total cost $5.6126 \times 10^{6}$ USD is chosen as the final optimal solution, including the CS plan \#4 and the network \#6 (i.e. line 5-6, 7-8, 12-13, 8-21 are determined as the norm-open tie lines, as red dotted lines in Fig. 10). The detailed CS deployment result is shown in Fig. 10, where the site of the CSs is indicated by the green box while the number of the corresponding charging devices is listed in the neighboring boxes. 


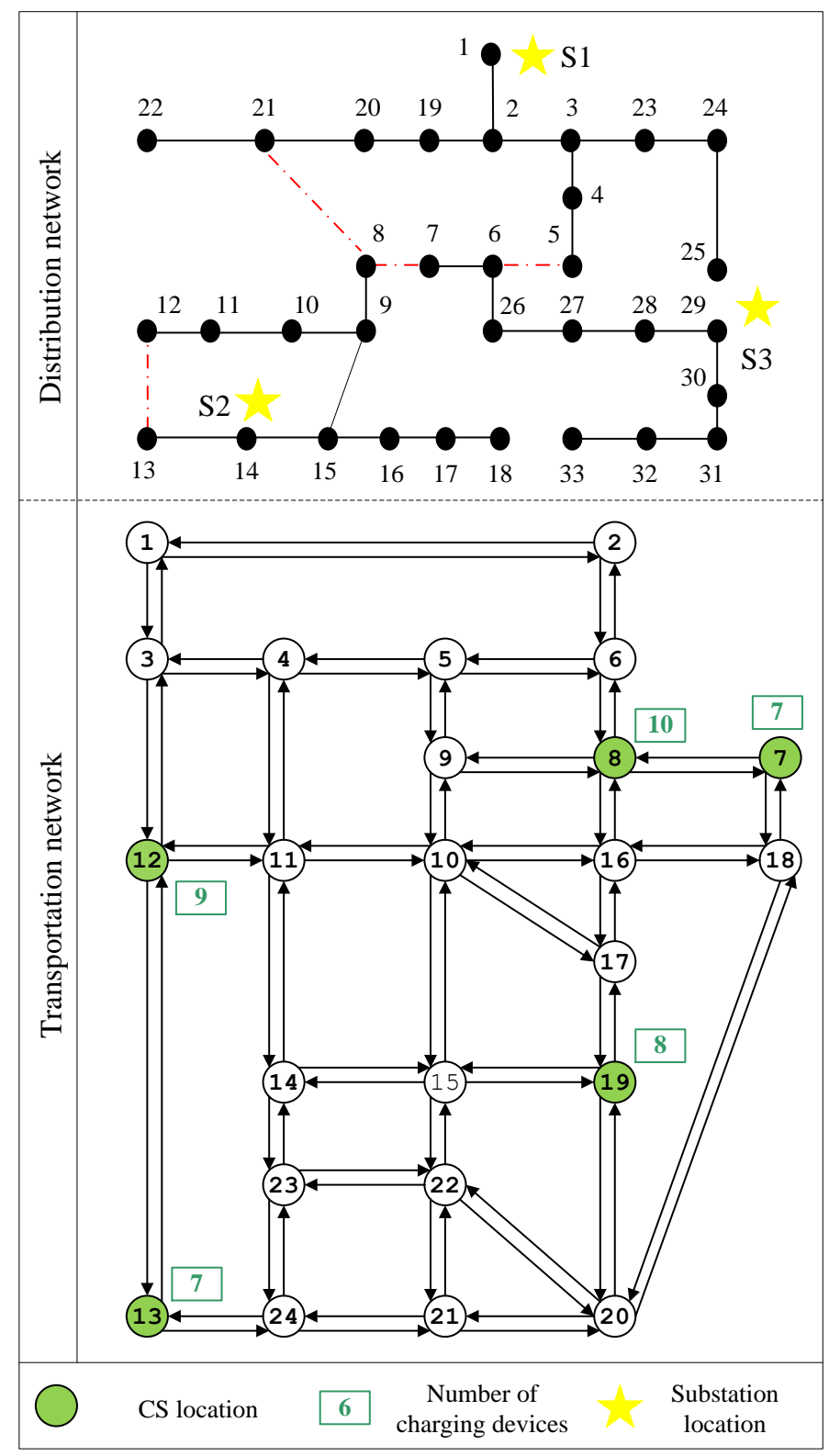

Fig. 10 Final optimal plan

Compared with the result obtained by the method in [21], whose final plan includes CS plan \#15 and the network \#3, and could survive only in a typical load profile condition, i.e. the topology of network \#3 cannot support any CS deployments in the load capability assessment according to the ten scenarios produced by the load templates, the final plan in this paper satisfies all the constraints from the coupled networks and pass the load capability constraints with the ten scenarios produced by the load templates, which indicate the proposed planning framework can greatly incorporate the uncertain operational conditions with the planning and make the solution more convincing.

On the other hand, the energy production and loss of the twelve available plans, i.e. the annual average power generation and the annual average power loss could be normalized formulated and presented as shown in Fig. 11. 


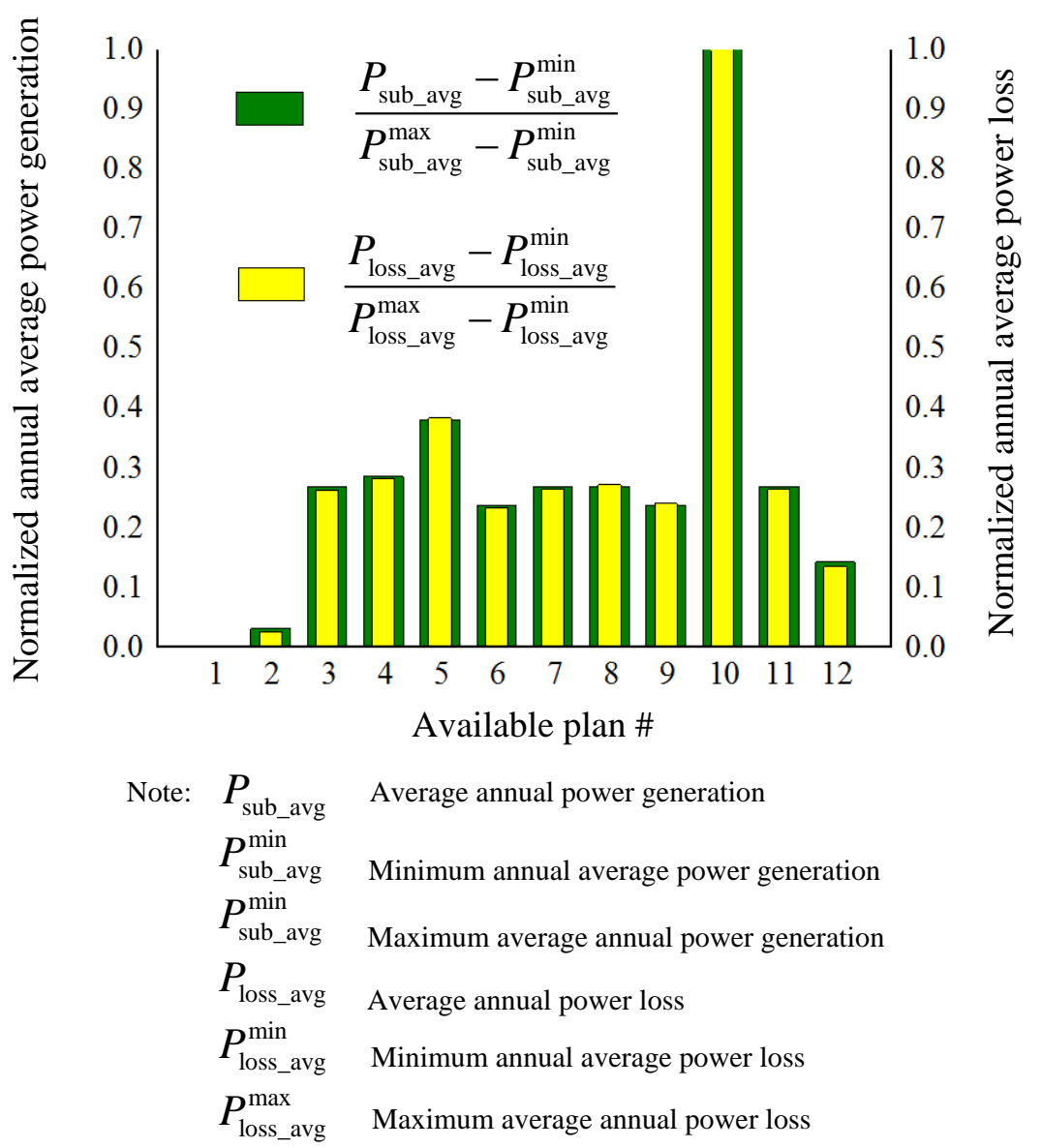

Fig. 11 Energy production and loss comparision of the available plans

It is clear to see from Fig. 11 that plan \#1 is with the minimum energy production and loss level. The reason why plan \#1 is not determined as the final optimal one is its corresponding annual investment for CSs is larger than that of plan \#2, as shown in Table 4, so the total cost. So, from the view of achieving the most economic objective in this paper, plan \#2 is the final optimal decision. Actually, the energy loss of plan \#2 is much lower than other majority plans, which would further prove that the most economic plan based on the proposed model owns higher energy efficiency and lower energy loss.

\subsection{Extended analysis}

According to the simulation results, we can also get some additional significant conclusions. The planning result aiming at achieving minimum economic cost can be selected from the candidate plans satisfying the load constraint. We can also analyze and assess the performance of the candidate plans in different views. Here the daily captured traffic flow (CTF) index is used to present the benefit from the utilization of the CSs, and formulated as:

$$
F_{T}=D \sum_{j \in \Omega} \sum_{t \in T} f n_{j, t} x_{j}^{\mathrm{CS} \_\mathrm{T}}\left(x_{j^{*}}^{\mathrm{CS}}\right)
$$

The details about the CTF on each candidate plan are shown in Fig. 12. Higher CTF indicates the plan with higher utilization of the CSs. As shown in Fig. 12, the CTF of 
the final plan \#2 by the framework in this paper is 3620.7 , which is not the maximum. The CTF just cares about the traffic flow condition and the geography of the area, in which the distribution network is barely considered. So in this purpose, plan \#10, would be the available solutions to achieve maximum CTF (the utilization of CSs). However, the cost of plan \#10 is still larger than plan \#2.

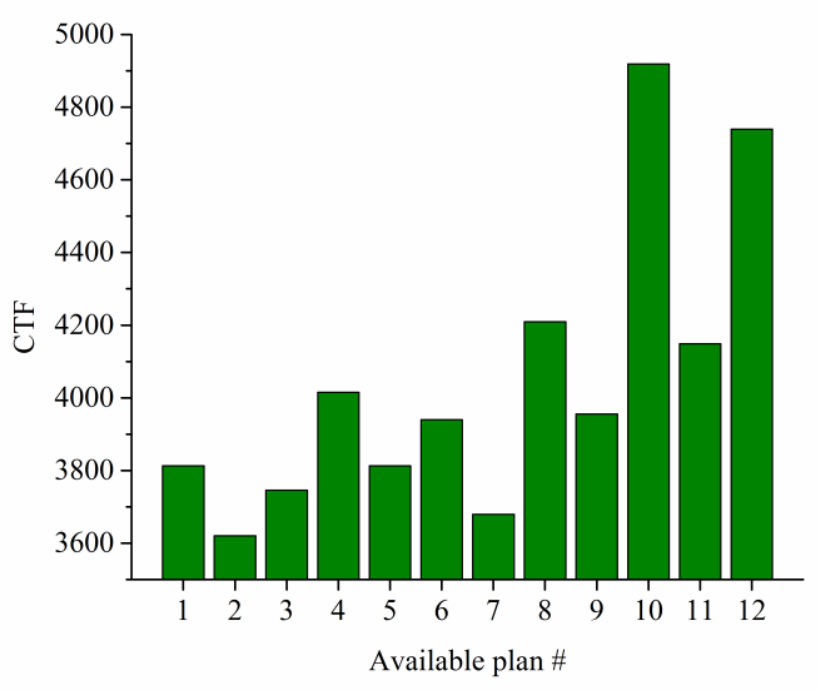

Fig. 12 The captured traffic flow in candidate plans

The CTF index can be used to reflect the "energy efficiency" in the transportation aspect to an extent. If both the economic and CTF objectives are adopted as the factors for determining the optimal planning result, the integrated model can be formulated as:

$$
\left\{\begin{array}{l}
F_{C} \rightarrow \min \\
F_{T} \rightarrow \max
\end{array}\right.
$$

Equation (27) is an optimal multi-objective formulation. Each objective has its own expected value and optimal trend. For the sake of different order of magnitudes of the two objectives, normalization should be taken firstly, which is formulated as:

$$
F_{i, j}^{\mathrm{N}}(x)=\frac{F_{i, j}(x)-F_{i}\left(x^{*}\right)}{F_{i}^{\mathrm{W}}-F_{i}\left(x^{*}\right)}
$$

Then, a game-theoretical decision method is introduced. A super criterion also known as the Bargaining function [38] is applied to compare the relative efficiencies of various multi-objectives, formulated as:

$$
\operatorname{Max} B I_{j}=\prod_{i=1}^{R}\left(1-F_{i, j}^{\mathrm{N}}(x)\right)^{\tau_{i}} \quad j=1, \ldots, N_{\mathrm{p}}
$$

where Eq. (29) is the bargaining function used to describe the distance from the solution point to the one with all worst value of the objectives.

Taken the Eq. (27) as the optimal objective, based on Eqs. (28)-(29), the bargaining function value of the available plans can be plotted in Fig. 13. 


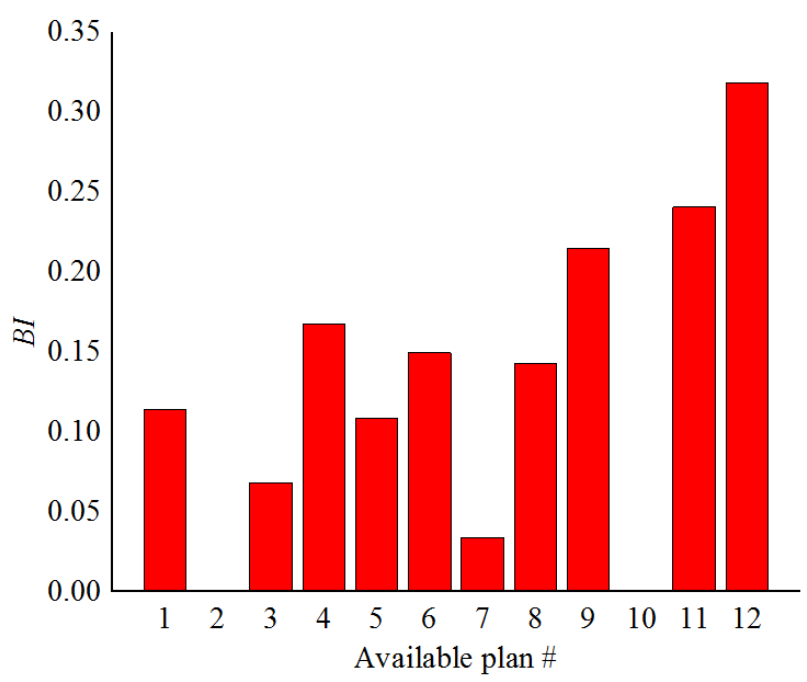

Fig. 13 Bargaining function value of each available plan

As seen from Fig. 13, though the economy of the plan \#2 is the best, but its CTF is the lowest, so the Bargaining function value is 0 . So the same result (0) is with plan \#10 with the highest CTF but largest economic cost. Plan \#12 has the largest Bargaining function value with the best equilibrium performance reflected by the total economic cost and the CTF. So it would be the final optimal planning result if the multi-objective model in Eq. (27) is utilized.

Except the CTF to reflect the energy efficiency in the transportation aspect, other indices, such as the reliability of the whole system, could be integrated to achieve the CS planning. Different objectives can lead to different solutions, since the economic objective is the basic and essential factor in the planning, so it is mainly proposed to be the focus in this paper to help guide CS planning. If more objectives are considered from different views, the multi-objective model can be applied as well.

\section{Conclusion}

This paper provides a novel planning framework for determining the siting and sizing of CSs coupling the interactions between the distribution and transportation networks. The capacity of the CSs is determined by the queuing model, in which the average arrival rate is formulated by the equilibrium traffic flow. Besides, load capability constraints are introduced to evaluate the plans including the CS and the tie line deployments, considering different operation scenarios aggregated by load profile templates. In this way, the static planning can be greatly integrated with the dynamic operation to make the placement of the CSs adapt to different operational conditions of the distribution network. The feasibility of the method is demonstrated and verified by the test case. Such temporal and locational methods can effectively guide EV charging infrastructures planning without violating the constraints from both the power and transportation systems.

The model and algorithms used in the planning framework are generic for the coupled networks to deploy the CSs, as well as the utilization of the load profile templates. Although the UK load templates are used in the test cases, load profile templates in 
other countries or districts could be also integrated in the proposed planning framework for optimally plan CSs in its specific target planning district, together with the transportation and power network information and the typical OD data in the target district. If there is no existing load templates in the specific country or district, they can be firstly generated by the clustering method with its historical load profiles, which is investigated by our research group and provided in [29]. It should be also noted that, the methodology presented in this paper mainly deploys the planning of the CSs from the economic objective view, future work would incorporate the distribution network expansion, aiming at achieving the coordinated planning as well.

\section{Funding}

This work was supported by National Science Foundation of China (51377111).

\section{References}

1. L. Zhou, F. Li, C. Gu, et al., "Cost/benefit assessment of a smart distribution system with intelligent electric vehicle charging," IEEE Transactions on Smart Grid, vol. 5, no. 2, pp: 839-847, 2014.

2. J. Liu, Y. Xiang, Wei Yang, et al., "Key technology analysis and study for charging and swapping service network and its coordinated planning with distribution network," Electric Power Construction, vol. 36, no. 7, pp: 61-68, 2015.

3. L. Dickerman, J. Harrison, "A new car, a new grid," IEEE Power and Energy Magazine, vol. 8, no. 2, pp: 55-61, 2010.

4. V. Boicea, "Energy storage technologies: the past and the present," Proceedings of the IEEE, vol. 102, no. 11, pp: 1777-1794, 2014.

5. B. Han, E. Bompard, F. Profumo, and Q. Xia, "Paths toward smart energy: a framework for comparision of the EU and China Energy Policy," IEEE Transactions on Sustainable Energy, vol. 5, no. 2, pp: 423-433, 2010.

6. General Office of the State Council of China, Guide for fast planning of the electric vehicle charging infrastructures, Available online: http://www.gov.cn/zhengce/content/2015-10/09/ content_10214. htm.

7. E. Iversen, J. Morales, H. Madsen, "Optimal charging of an electric vehicle using a Markov decision process," Applied Energy, vol. 123, pp:1-12, 2014.

8. Y. Xiang, J. Liu, and Y. Liu, "Optimal active distribution system management with aggregated plug-in electric vehicle," Electric Power Systems Research, vol. 131, pp: 105-115, 2016.

9. Y. Mu, J. Wu, N. Jenkins, H. Jia, C. Wang, "A spatial-temporal model for grid impact analysis of plug-in vehicles," Applied Energy, vol. 114, pp:456-465, 2014.

10. W. Yang, H. Zhou, J. Liu, et al., "Market evolution modeling for electric vehicles based on system dynamics and multi-Agents," 2015 International Symposium on Smart Electric Distribution Systems and Technologies (EDST), CIGRE, Wien, Austria, pp: 1-6, Sept. 2015.

11. X. Tang, J. Liu, et al., "Electric vehicle charging station planning based on computational geometry method," Automation of Electric Power Systems, vol. 36, no. 8, pp: 24-30, 2012.

12. S. Guo, H. Zhao, "Optimal site selection of electric vehicle charging station by using fuzzy TOPSIS based on sustainability perspective," Applied Energy, vol. 158, pp:390-402, 2015.

13. A. Lam, Y. Leung, and X. Chu, "Electric vehicle charging station placement: formulation, complexity and solutions," IEEE Transactions on Smart Grid, vol. 5, no. 6, pp: 2846-2856, 2014.

14. P. Sadeghi-Barzani, A. Rajabi-Ghahnavieh, H. Kazmi-Karegar, "Optimal fast charging station placing and sizing," Applied Energy, vol. 125, pp:289-299, 2014.

15. I. Safak Bayram, A. Tajer, M. Abdallah, K. Qaraqe,"Capacity Planning Frameworks for Electric Vehicle Charging Stations With Multiclass Customers," IEEE Transactions on Smart Grid, vol.6, no.4, pp:608-617, 2015.

16. H. Zhang, Z. Hu, Z. Xu, Y. Song, "An integrated planning framework for different types of PEV charging facilities in urban area," IEEE Transactions on Smart Grid, to be published, DOI: 10.1109/ TSG.2015.2436069. 
17. M. Cruz-Zambrano, C. Corchero, et al., "Optimal location of fast charging stations in Barcelona: a flow-capturing approach," $201310^{\text {th }}$ International Conference on the European Energy Market, pp: 1-6, Stockholm, 27-31 May, 2013.

18. S. Yang, M. Wu, X. Yao, J. Jiang, "Load modeling and identification based on ant colony algorithms for EV charging stations," IEEE Transactions on Power System, vol. 30, no. 4, pp:1997-2003, 2015.

19. G. Wang, Z. Xu, et al., "Traffic-constrained multi-objective planning of electric vehicle charging stations," IEEE Transactions on Power Delivery, vol. 28, no. 4, pp: 2363-2372, 2013.

20. L. Zhang, B. Shaffer, T. Brown, G. Samuelsen, "The optimization of DC fast charging deployment in California," Applied Energy, vol. 157, pp: 111-122, 2015.

21. Y. Xiang, J. Liu, et al., "A traffic flow based planning strategy for optimal siting and sizing of charging stations," in IEEE PES 2015 Asia-Pacific Power and Energy Conference, 15-18 Nov. 2015, pp: 1-5, Brisbane, Australia.

22. L. Suo, W. Tang, M. Bai, L. Zhang, "Locating and sizing of centralized charging stations in distribution network considering load shifting," Proceedings of the CSEE, vol. 34, no. 7, pp: 1052-1060, 2014.

23. C. Gao, and L. Zhang, F. Xue, et al., "Consider centralized charging station addressing points of power grid planning study," Proceedings of the CSEE, vol. 32, no. 7, pp: 40-46, 2012.

24. S. Feng, H. Gao, C. Guo, "Evaluation of structural types of urban road network," Journal of Harbin Institute of Technology, vol. 39, no. 10, pp: 1610-1613, 2007.

25. Cloud BaiduMap API, Available: http:// developer.baidu.com/ map/lbs-cloud.htm.

26. Y. Sheffi., Urban transportation network: equilibrium analysis with mathematical programming methods, Prentice Hall, 1985.

27. Y. Zeng, L. Dong, J. Ma, Queuing modeling, analysis and simulation, Xi'an University of Electronic Science and Technology Press, 2011.

28. W. Yang, H. Zhou, J. Liu, et al., "Market evolution modeling for electric vehicles based on system dynamics and multi-Agents," in 2015 International Symposium on Smart Electric Distribution Systems and Technologies (EDST), CIGRE, Wien, Austria, pp: 1-6, Sept. 2015.

29. R. Li, Load profiling on time and spectral domain: from big data to smart data, University of Bath, 2014.

30. R. Li, C. Gu, F. Li, et al., "Development of low voltage network templates - Part II peak load estimation by cluster wise regression: peak load estimation by cluster wise regression," IEEE Transactions on Power Systems, vol. 30, no. 6, pp: 3045-3052, 2015.

31. S. Allera, "Load profiling for energy trading and settlements in the UK electricity markets," in DistribuTECH Europe DA/DSM, London, UK, 1998.

32. E. Miguez, J. Cidras, et al., "An improved branch-exchange algorithm for large-scale distribution network planning," IEEE Transactions on Power Systems, vol. 17, no. 4, pp: 931-936, 2002.

33. ELEXON, Load profile classes, Available: http:// ELEXON.co.uk.

34. Sioux-Falls network, Available: http://www.bgu.ac.il/ bargera /tntp/.

35. Q. Guo, S. Xin, et al., "Rapid-charging navigation of electric vehicles based on real-time power systems and traffic data," IEEE Transactions on Smart Grid, vol. 5, no. 4, pp: 1969-1979, 2014.

36. Metropolitan, Household Characteristics, Available online: http://www.metrocouncil.org/ Transportation/Publications-And-Resources/TBI2000HouseholdCharacteristics13County-pdf.aspx.

37. H. Zhang, Z. Hu, Y. Song, et al., "A prediction method for electric vehicle charging load considering spatial and temporal distribution”, Automation of Electric Power Systems, vol. 38, no. 1, pp. 13-20, 2014.

38. C. Huang, and Y. Huang, "Combined wavelet-based networks and game-theoretical decision approach for real-time power dispatch," IEEE Transactions on Power Systems, vol. 17, no. 3, pp: 633-639, 2002. 